\title{
RELATIVISTIC MEAN FIELD STUDY OF SM ISOTOPES WITH FTBCS APPROACH
}

\author{
Afaque Karim ${ }^{1}$,Tasleem A. Siddiqui ${ }^{1}$, and Shakeb Ahmad ${ }^{1}$ \\ ${ }^{1}$ Department of Physics, Aligarh Muslim University, Aligarh, INDIA
}

\begin{abstract}
A theoretical study of the thermal pairing correlation as a function of temperature is performed for eveneven ${ }^{148-154}$ Sm isotopes using Finite Temperature Bardeen-Cooper-Schrieffer (FTBCS) approach within the Relativistic Mean Field (RMF) model. Numerical results obtained at $T=0$ are found to be consistent with the available experimental values. Further, results show the thermal dependency of various nuclear

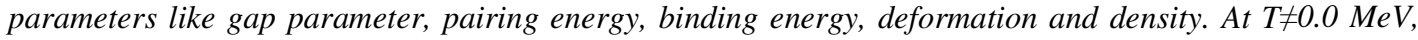
the destruction of Cooper pairs and the pairing phase transition as well as shape transition is observed in ${ }^{148-154} \mathrm{Sm}$ nuclei at critical temperature $T_{c} \neq 0$.
\end{abstract}

\section{KEYWORDS}

Relativistic Mean Field Theory, FTBCS, Pairing Correlation, Binding Energy, Gap Parameter

\section{INTRODUCTION}

It is well established that the pairing correlation effect play quite an important role in the determination of most of the ground state physical properties of a nuclear system. The nuclear structure, being the Fermionic system, exhibits many similarities with the atomic structure of materials. Among materials, superconductors are familiar to us. Moreover, it is well known that the pair correlations in macroscopic superconductors are destroyed by increasing the temperature or external magnetic field. At finite temperature metal superconductors undergoes a phase transition [1]. However, finite many-body systems also exhibit a phase transitional behavior, but the effect of super fluid-to-normal phase transition due to the breaking and restoration of pairing within a Fermionic system remains an open question. The critical field decides the boundary between the superfluid phase and the normal phase, which is a decreasing function of the temperature $T$. The standard theory which describes this phase transitional regime very accurately is the Bardeen-Cooper-Schrieffer (BCS) theory. BCS theory is a mean-field theory with a grandcanonical ensemble approach.

First relevant theory, which considers the pairing correlation in nuclear many-body system, was proposed by Bohr and Mottelson, and Pines in 1958 [2]. Also, analysis of certain nuclear properties such as, the suppression of the moments of inertia of rotating nuclei and the observed energy gaps give quite clear evidence, that the pairing correlation is essential for describing atomic nuclei $[3,4]$. Further, it is well established that certain symmetry violations in the BCS approach may imply considerable effects while calculating various ground state nuclear properties of a finite Fermi systems, such as atomic nuclei [5-12]. But most of these nuclear properties are studied at zero temperature $T=0$. So, the study of nuclear properties at non zero temperature $T \neq 0$ has been important subject of research from a long time [13, 14]. Theoretically, DOI : 10.14810/ijrap.2015.4405 
International Journal of Recent advances in Physics (IJRAP) Vol.4, No.4, November 2015

different models have been reported in connection with temperature dependent mean-field approach $[15,16]$. The other important thing is the method employed to solve the pairing interaction. The pairing field has been primarily studied in BCS or the Hartree-Fock-Bogoliubov (HFB) approximation. The microscopic description of pairing effects at finite temperature has obvious advantages of unified picture. In this, the pairing field depicts a sudden transitional behavior as a function of rotational frequency and temperature [17, 18]. At a certain rotational frequency or temperature, the pairing correlations are found to be finite. Later above the translational point, these correlations vanishes quickly. Thus, the projection at finite temperature is more important part as it leads to study of more realistic models.

The purpose of present work is to study the effect of pairing correlation on various ground state properties of atomic nuclei at finite temperature within the conventional Finite Temperature Bardeen-Cooper-Schrieffer (FTBCS) approach, i.e. temperature dependency of gap parameter [17-23]. We take into account the thermal occupancy of quasi particles. These are basically fermions . This FTBCS pairing is introduced in the Relativistic Mean Field (RMF) model [24-26] to study various nuclear parameters. Numerical calculations are performed for even-even Samarium (Sm) nuclei, and mainly stable isotopes of Samarium ${ }^{148-154} \mathrm{Sm}$ nuclei.

Even-Even Samarium (Sm) isotopes have been the focal point of large number of experimental studies in the past [27-32]. The study of Samarium nuclei has been a challenging as well as an interesting theoretical problem. The Samarium nuclei lie in the range from near spherical to well deformed shapes. ${ }^{148} \mathrm{Sm}$ is believed to be basically spherical while ${ }^{154} \mathrm{Sm}$ is thought to be well deformed nucleus and ${ }^{150-152} \mathrm{Sm}$ is transitional nuclei [33]. In the present work ${ }^{148-154} \mathrm{Sm}$ nuclei are studied within the RMF model along with FTBCS approach for pairing correlation treatment to get various nuclear parameters.

The paper is organized as follows. In section 2, theoretical formalism is presented which includes a brief description RMF formalism, and FTBCS approach for pairing correlation treatment. Sec 3 presents the numerical results of our calculations along with its description. Sec 4 summarizes and concludes our results.

\section{FORMALISM}

\subsection{RMF Formalism}

The model is defined by the standard Lagrangian density [24-26]

$$
\begin{aligned}
\mathcal{L}=\bar{\Psi}\left(i \gamma_{\mu} \partial^{\mu}-\right. & M) \Psi+\frac{1}{2}\left(\partial_{\mu} \sigma \partial^{\mu} \sigma-m_{\sigma}^{2} \sigma^{2}\right)-U(\sigma)-\frac{1}{4} \boldsymbol{\Omega}_{\mu \nu} \boldsymbol{\Omega}^{\mu \nu}+\frac{1}{2} m_{\omega}^{2} \omega_{\mu} \omega^{\mu}-\frac{1}{4} \overrightarrow{\boldsymbol{R}}_{\mu \nu} \overrightarrow{\boldsymbol{R}}^{\mu \nu} \\
& +\frac{1}{2} m_{\rho}^{2} \vec{\rho}_{\mu} \vec{\rho}^{\mu}-\frac{1}{4} \boldsymbol{F}_{\mu \nu} \boldsymbol{F}^{\mu \nu}-g_{\sigma} \bar{\Psi} \sigma \Psi-g_{\omega} \bar{\Psi} \gamma_{\mu} \omega^{\mu} \Psi-g_{\rho} \bar{\Psi} \gamma_{\mu} \vec{\tau} \vec{\rho}^{\mu} \Psi \\
& -e \frac{1+\tau_{3}}{2} \bar{\Psi} \gamma_{\mu} \boldsymbol{A}^{\mu} \psi
\end{aligned}
$$

where $\mathrm{M}$ is the bare nucleon mass and $\psi$ denotes the Dirac spinors. The masses $m_{\sigma}, m_{\omega}$, and $m_{\rho}$ are those of the $\sigma$-meson, $\omega$-meson, and the $\rho$-meson, and $g_{\sigma}, g_{\omega}, g_{\rho}$, are coupling constants for the mesons to the nucleons respectively, e is the charge of the proton, and $\vec{\tau}$ the iso-spin operator. These coupling constants and unknown meson masses are the Lagrangian eqn.(1) parameters. Here, $\Omega^{\mu \nu}, \vec{R}^{\mu \nu}$, and $F^{\mu \nu}$ are the field tensors of the vector fields $\omega, \rho$, and the photon: 
$\boldsymbol{\Omega}^{\mu \nu}=\partial^{\mu} \omega^{v}-\partial^{v} \omega^{\mu}$

$\overrightarrow{\boldsymbol{R}}^{\mu \nu}=\partial^{\mu} \vec{\rho}^{v}-\partial^{v} \vec{\rho}^{\mu}$

$\boldsymbol{F}^{\mu \nu}=\partial^{\mu} \boldsymbol{A}^{v}-\partial^{v} \boldsymbol{A}^{\mu}$

In the above expressions, isovectors are denoted by arrows, and bold-faced symbols are used for vectors in ordinary three-dimensional space. The functions $g_{\sigma}, g_{\omega}, g_{\rho}$, are assumed to be vertex functions of Lorentz-scalar bilinear forms of the nucleon operators. For a realistic description of complex nuclear system properties a non-linear self-coupling $U(\sigma)$ of the $\sigma$-meson,

$U(\sigma)=\frac{1}{3} g_{2} \sigma^{3}+\frac{1}{4} g_{3} \sigma^{4}$

has been used which turned out to be crucial. From the above Lagrangian we obtain the field equations for the nucleons and mesons. These equations are solved by expanding the above and lower component of Dirac spinors and the boson fields in an axially deformed harmonic oscillator basis with an initial deformation $\beta_{0}$. The set of coupled equation is solved numerically by a self consistent iteration method. The center of mass energy correction is estimated by the usual harmonic oscillator formula

$$
E_{c m}=\frac{3}{4}\left(41 A^{-1 / 3}\right)
$$

Table 1. The parameters of the NL3* parameterization in the Lagrangian.

\begin{tabular}{ll}
\hline Parameter & $\mathrm{NL3}{ }^{*}[34]$ \\
\hline $\mathbf{m}$ & 939 \\
$\mathbf{m}_{\boldsymbol{\sigma}}$ & 502.5742 \\
$\mathbf{m}_{\boldsymbol{\omega}}$ & 782.600 \\
$\mathbf{m}_{\boldsymbol{\rho}}$ & 763.000 \\
$\mathbf{g}_{\boldsymbol{\sigma}}$ & 10.0944 \\
$\mathbf{g}_{\boldsymbol{\omega}}$ & 12.8065 \\
$\mathbf{g}_{\boldsymbol{\rho}}$ & 4.5748 \\
$\mathbf{g}_{\mathbf{2}}$ & -10.8093 \\
$\mathbf{g}_{3}$ & -30.1486 \\
\hline
\end{tabular}

The quadruple deformation is evaluated from the resulting proton and neutron quadruple moments as

$$
Q=Q_{n}+Q_{p}=\sqrt{\frac{16 \pi}{5}}\left(\frac{3}{4 \pi} A R^{2} \beta_{2}\right)
$$

The root mean square (rms) matter radius defined as $\left\langle r_{m}^{2}\right\rangle=\frac{1}{A} \int \rho\left(r_{\perp}, z\right) r^{2} d \tau$,

where $\mathrm{A}$ is the mass number, and $\rho\left(r_{\perp}, z\right)$ is the deformed density. The total binding energy (B.E.) and other observables are also obtained using the standard relations, given in Refs. 25 and 26 . The present investigation uses the very successful interaction potential parameter set NL3* [34], given in Table 1, which is a modern version of the widely used interaction potential parameter set NL3 [35]. NL3* provides improved version of various ground state ,bulk as well as surface properties of nuclei over NL3 parameter. Moreover, It provides extremely good description of excited in spherical as well as in deformed nuclei. However, in the mean time, several other RMF 
International Journal of Recent advances in Physics (IJRAP) Vol.4, No.4, November 2015

interactions have been developed. In particular, the density dependent meson-exchange DDME1[36] and DD-ME2 [37] effective interaction. These effective interactions have been adjusted to improve the isovector channel, which has been weak point of NL3 effective interaction, and provide a very successful description of different aspects of finite nuclei $[38,39]$. Recently, a new DD-PC1 [40](density-dependent point coupling) effective interaction has been developed, which work very well in deformed nuclei.

\subsection{BCS Pairing at Finite Temperature (FTBCS)}

The pairing correlations have been included for realistic calculation of medium and heavy nuclei. In principle, the microscopic Hartree-Fock-Bogoliubov (HFB) theory should be used, which have been developed in several papers [38-42] and the references given therein. However, BCS pairing approach is a simpler approach with constant gap and is found to be reasonably well for pairing calculations of a wide range of nuclei which are not too far from the $\beta$ stability line. But this simple approach fails for nuclei far from the valley of $\beta$ stability where the coupling to the continuum is important [43]. Many groups as well as we have treated pairing correlations using BCS approach in earlier studies [44-46]. The BCS approach generally fails for light neutron rich nuclei. Here, we have not considered light neutron rich nuclei. Thus, the RMF results with this treatment are reliable. But, In order to get a better picture of the states we reformulate the BCS theory. We introduce new operators instead of creation and annihilation operators of the nuclear states by unitary transformation, which will turn out to be the creation and annihilation operators of the quasiparticles of superconducting state. This can be achieved most simply in a mean field approach by self consistently calculating the non-vanishing anomalous averages of creation and annihilation operators at finite temperature as thermal averages, neglecting their fluctuations. The mean field Hamiltonian takes the form as

$$
\begin{gathered}
H_{B C S}=-\sum_{k, k^{\prime}} V_{k, k^{\prime}}\left\langle c_{k}^{\dagger} c_{\bar{k}}^{\dagger}\right\rangle\left\langle c_{\bar{k}^{\prime}} c_{k^{\prime}}\right\rangle+\sum_{k} \epsilon_{k}\left(c_{k}^{\dagger} c_{k}+c_{\bar{k}}^{\dagger} c_{\bar{k}}\right)-\sum_{k}\left(\Delta_{k} c_{k}^{\dagger} c_{\bar{k}}^{\dagger}\right. \\
\left.+\Delta_{k^{*}} c_{\bar{k}} c_{k}\right) .
\end{gathered}
$$

with a temperature dependent order parameter defined as

$\Delta_{k}=-\sum_{k^{\prime}} V_{k, k^{\prime}}\left\langle c_{\bar{k}^{\prime}} c_{k^{\prime}}\right\rangle$

$\Delta_{k^{*}}=-\sum_{k^{\prime}} V_{k^{\prime}, k}\left\langle c_{k^{\prime}}^{\dagger} c_{k^{\prime}}^{\dagger}\right\rangle$

The Hamiltonian can be diagonalized by repeating formally the same steps that were made at $\mathrm{T}=0$. The creation and annihilation operators of the quasiparticles are defined in terms of the creation and annihilation operators of nucleonic states by unitary transformation.

$$
\begin{aligned}
& \alpha_{k}=u_{k} c_{k}-v_{k} c_{\bar{k}}^{\dagger} \\
& \alpha_{k}^{\dagger}=u_{k}^{*} c_{k}^{\dagger}-v_{k}^{*} c_{\bar{k}} \\
& \alpha_{\bar{k}}=u_{k} c_{\bar{k}}+v_{k} c_{k}^{\dagger} \\
& \alpha_{\bar{k}}^{\dagger}=u_{k}^{*} c_{\bar{k}}^{\dagger}+v_{k}^{*} c_{k}
\end{aligned}
$$


The inverse transformation is

$$
\begin{aligned}
& c_{k}=u_{k}^{*} \alpha_{k}+v_{k} \alpha_{\bar{k}}^{\dagger} \\
& c_{k}^{\dagger}=u_{k} \alpha_{k}^{\dagger}+v_{k}^{*} \alpha_{\bar{k}} \\
& c_{\bar{k}}=u_{k}^{*} \alpha_{\bar{k}}-v_{k} \alpha_{k}^{\dagger} \\
& c_{\bar{k}}^{\dagger}=u_{k} \alpha_{\bar{k}}^{\dagger}-v_{k}^{*} \alpha_{k}
\end{aligned}
$$

The Hamiltonian takes the diagonal form

$$
H=E(T)+\sum_{k} E_{k}\left(\alpha_{k}^{\dagger} \alpha_{k}+\alpha_{\bar{k}}^{\dagger} \alpha_{\bar{k}}\right) .
$$

with quasi particle energy $E_{k}$ and remaining temperature dependent part $E(T)$.

Here,

$$
E_{k}=\sqrt{\epsilon_{k}^{2}+\left|\Delta_{k}\right|^{2}}
$$

And

$$
E(T)=-\sum_{k, k^{\prime}} V_{k, k^{\prime}}\left\langle c_{k}^{\dagger} c_{\bar{k}}^{\dagger}\right\rangle\left\langle c_{\bar{k}^{\prime}} c_{k^{\prime}}\right\rangle+2 \sum_{k} \epsilon_{k}\left|v_{k}\right|^{2}-\sum_{k}\left(\Delta_{k} u_{k} v_{k}^{*}+\Delta_{k}^{*} u_{k}^{*} v_{k}\right) .
$$

The temperature dependence of $\Delta_{k}$ results in the temperature dependence of the quasiparticle energy that are determined self-consistently. Combining eqn. (7) and eqn.(8) with eqns. (13), (14), (15) and eqn. (16) we have

$\Delta_{k}=-\sum_{k^{\prime}} V_{k, k^{\prime}}\left\langle\left(u_{k^{\prime}}^{*} \alpha_{\bar{k}^{\prime}}-v_{k^{\prime}} \alpha_{k^{\prime}}^{\dagger}\right)\left(u_{k^{\prime}}^{*} \alpha_{k^{\prime}}+v_{k^{\prime}} \alpha_{\bar{k}^{\prime}}^{\dagger}\right)\right\rangle$.

We assume usually that normal averages are finite for the quasiparticles.Thus, we get

$\Delta_{k}=-\sum_{k^{\prime}} V_{k, k^{\prime}} u_{k^{\prime}}^{*} v_{k^{\prime}}\left(\left\langle\alpha_{\bar{k}^{\prime}} \alpha_{\bar{k}^{\prime}}^{\dagger}\right\rangle-\left\langle\alpha_{k^{\prime}}^{\dagger} \alpha_{k^{\prime}}\right\rangle\right)$.

Since the quasiparticles are fermions, their thermal occupation in thermodynamic equilibrium is given by Fermi distribution function at the quasiparticle energy.

$$
f_{0}\left(E_{k}\right)=\frac{1}{e^{\beta E_{k}+1}}
$$

The self consistency condition then leads to

$\Delta_{k}=-\sum_{k, k^{\prime}} V_{k, k^{\prime}} u_{k^{\prime}} v_{k^{\prime}}\left[1-2 f_{0}\left(E_{k}^{\prime}\right)\right]$.

In this way, we calculate the thermal dependence of gap parameter. 


\section{RESULTS AND DISCUSSIONS}

\subsection{Ground state properties using RMF+BCS approach}

There exist a number of parameter sets for solving the standard RMF Lagrangian. The ground state properties (i.e. at $\mathrm{T}=0.0 \mathrm{MeV}$ ) like the B.E., quadruple deformation parameters $\beta_{2}$, charge radii $r_{c h}$, and other bulk properties are evaluated by using relativistic parameter set at zero temperature. It is found that, more or less, the recent parameter produced the ground state properties for ${ }^{148-154} \mathrm{Sm}$ nuclei which is close to the experimental values, and, are showninTable 2 .

\subsection{Nuclear Parameter using RMF+FTBCS approach}

Temperature dependent variations $(\mathrm{T}=0.0 \mathrm{MeV}$ to $2.0 \mathrm{MeV})$ of the various nuclear parameters such as binding energy (B.E.), the quadrupole deformation parameter $\beta_{2}$, neutron gap parameter $\Delta_{n}$ and proton gap parameter $\Delta_{\boldsymbol{p}}$, and pairing energy $E_{\text {pair }}$ are calculated within the RMF+FTBCS approach for even-even $\mathrm{Sm}$ isotopes.

Table 2. The RMF (NL3 ${ }^{*}$ ) results for B.E, the quadrupole deformation parameter $\beta_{2}$, charge radii

$r_{c h}$ for even-even Sm isotopes compared with the experimental data wherever available.

\begin{tabular}{l|cll}
\hline Nucleus & Properties & Theoretical & Experimental $[47,48,49]$ \\
\hline${ }^{148} \mathrm{Sm}$ & B.E. $(\mathrm{MeV})$ & $\mathbf{1 2 2 5 . 7 3}$ & $\mathbf{1 2 2 5 . 4 0}$ \\
& $\beta_{2}$ & 0.14 & 0.13 \\
${ }^{150} \mathrm{Sm}$ & $r_{c h}$ & 5.00 & 5.01 \\
& B.E. $(\mathrm{MeV})$ & $\mathbf{1 2 3 9 . 6 0}$ & $\mathbf{1 2 3 9 . 2 5}$ \\
& $\beta_{2}$ & 0.21 & 0.19 \\
& $r_{c h}$ & 5.04 & 5.04 \\
${ }^{152} \mathrm{Sm}$ & B.E. $(\mathrm{MeV})$ & $\mathbf{1 2 5 3 . 9 8}$ & $\mathbf{1 2 5 3 . 1 1}$ \\
& $\beta_{2}$ & 0.31 & 0.29 \\
& $r_{c h}$ & 5.08 & 5.09 \\
${ }^{154} \mathrm{Sm}$ & B.E. $(\mathrm{MeV})$ & $\mathbf{1 2 6 8 . 0 5}$ & $\mathbf{1 2 6 6 . 9 4}$ \\
& $\beta_{2}$ & 0.34 & 0.33 \\
& $r_{c h}$ & 5.11 & 5.12 \\
\hline
\end{tabular}

The calculated neutron gap parameter $\Delta_{n}$ and proton gap parameter $\Delta_{p}$ at zero temperature $(T=0)$ for ${ }^{148-154} \mathrm{Sm}$ nuclei using RMF+BCS is shown in Table 2. The gap parameter decreases with increasing mass number (A) confirms the shell structure, and that in case of heavier nuclei the outer shells are closer to each other. Figure 1 show the temperature dependent variation of neutron gap parameter $\Delta_{n}$ and proton gap parameter $\Delta_{p}$ with temperature for T=0.0 to $2.0 \mathrm{MeV}$ in RMF(NL3*)+FTBCS approach. In both the cases, we can see that both $\Delta_{n}$ and $\Delta_{p}$ decreases gradually with temperature. 


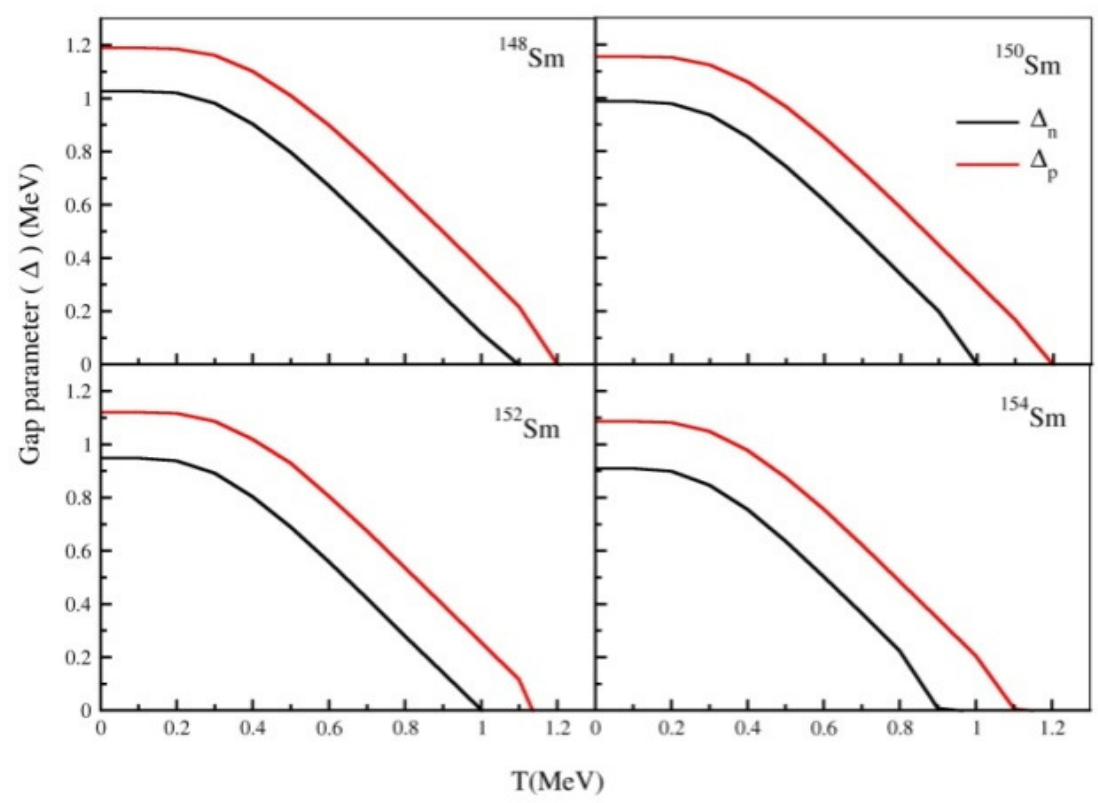

Figure 1. Variation of neutron and proton gap parameter with temperature for $\mathrm{T}=0.0$ to $2.0 \mathrm{MeV}$ using RMF(NL3*)+FTBCS approach for ${ }^{148-154} \mathrm{Sm}$.

Moreover, the decreasing trend of the gap parameter with temperature explains the fact that thermal energy can destroy the pairing of nucleons. The gap parameter in both the cases drops to zero at some finite value of temperature, which is known as the value of critical temperature for $\Delta_{n}$ and $\Delta_{\boldsymbol{p}}$, and is shown in Table 3 . This is the value where nuclei transits from superconducting state to normal state or one can termed it as a transition point for pairing phase transition in nuclei which is strongly dependent of temperature.

Table 2.Neutron gap parameter $\Delta_{\boldsymbol{n}}$ and Proton gap parameter $\Delta_{\boldsymbol{p}}$ at zero temperature ( $\mathrm{T}=0.0$ $\mathrm{MeV}$ ) for even-even Sm isotopes using RMF(NL3*)+FTBCS approach.

\begin{tabular}{|l|l|l|}
\hline Nucleus & $\Delta_{\boldsymbol{n}}(\mathrm{MeV})$ & $\Delta_{\boldsymbol{p}}(\mathrm{MeV})$ \\
\hline${ }^{148} \mathbf{S m}$ & 1.03 & 1.19 \\
\hline${ }^{150} \mathbf{S m}$ & 0.98 & 1.15 \\
\hline${ }^{152} \mathbf{S m}$ & 0.94 & 1.12 \\
\hline${ }^{154} \mathbf{S m}$ & 0.91 & 1.09 \\
\hline
\end{tabular}

Table 3.The critical temperature $T_{c}$ (in $\mathrm{MeV}$ ) for $\Delta_{n}$ and $\Delta_{p}$ for even-even $\mathrm{Sm}$ isotopes using RMF(NL3*)+FTBCS approach

\begin{tabular}{|c|c|c|}
\hline Nucleus & $\mathbf{T}_{\mathbf{c}}\left(\Delta_{n}\right)$ & $\mathbf{T}_{\mathbf{c}}\left(\Delta_{\boldsymbol{p}}\right)$ \\
\hline${ }^{\mathbf{1 4} 8} \mathbf{S m}$ & 1.1 & 1.19 \\
\hline${ }^{150} \mathbf{S m}$ & 1.0 & 1.20 \\
\hline${ }^{152} \mathbf{S m}$ & 1.0 & 1.13 \\
\hline${ }^{154} \mathbf{S m}$ & 0.9 & 1.11 \\
\hline
\end{tabular}


International Journal of Recent advances in Physics (IJRAP) Vol.4, No.4, November 2015

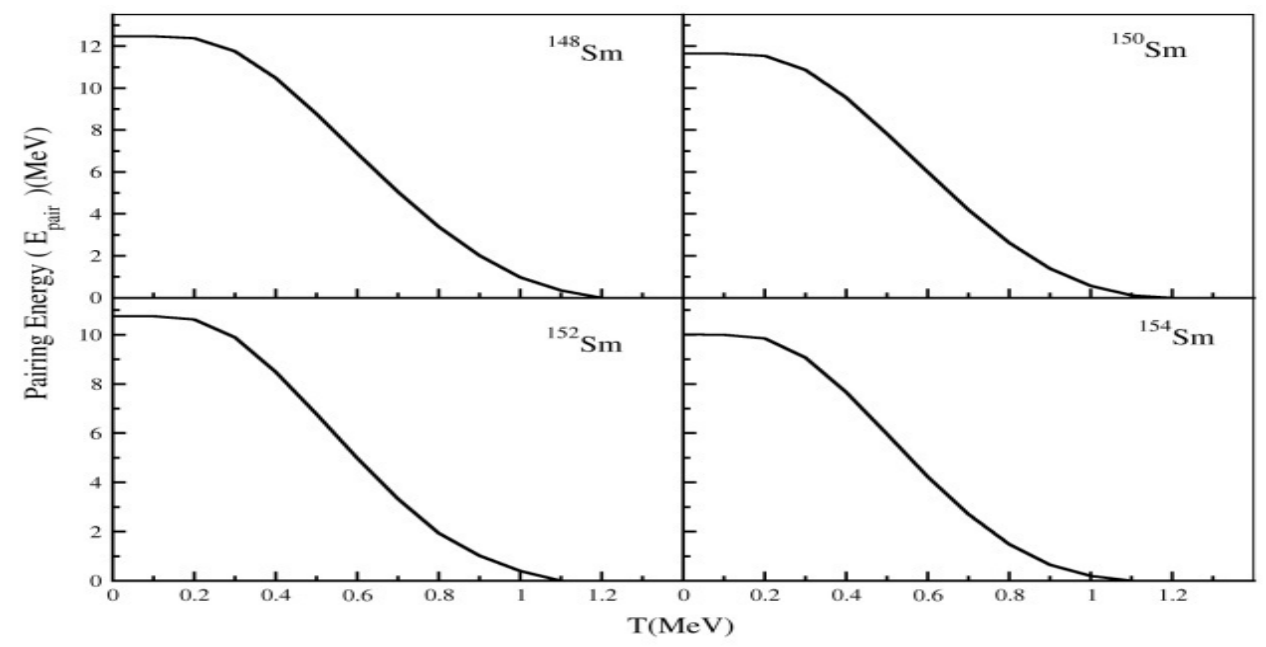

Figure 2. Variation of Pairing Energy with temperature for $\mathrm{T}=0.0$ to $2.0 \mathrm{MeV}$ using RMF(NL3*)+FTBCS approach for ${ }^{148-154} \mathrm{Sm}$

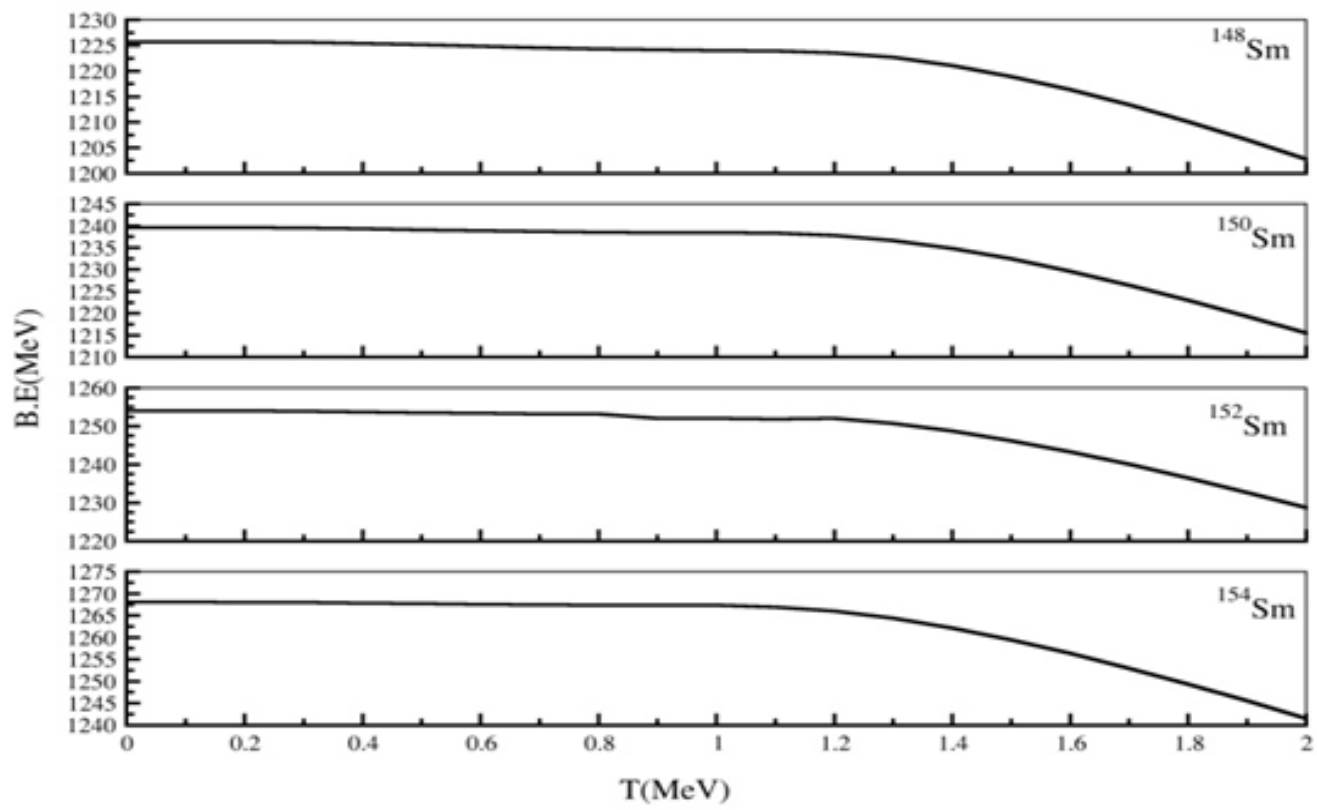

Figure 3. Variation of binding energy with temperature for $\mathrm{T}=0.0$ to $2.0 \mathrm{MeV}$ using RMF(NL3*)+FTBCS approach for ${ }^{148-154} \mathrm{Sm}$ 


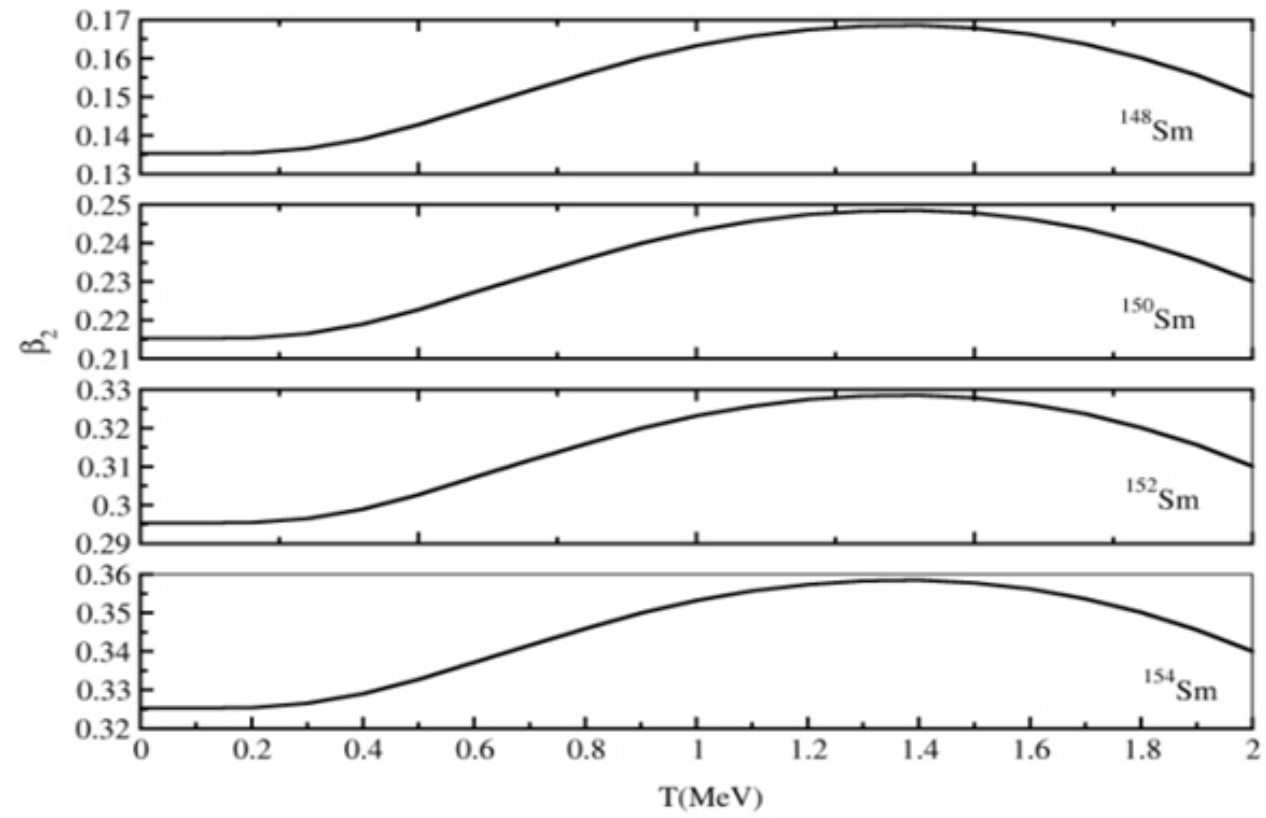

Figure 4. Variation of deformation parameter with temperature for $\mathrm{T}=0.0$ to $2.0 \mathrm{MeV}$ using RMF(NL3*)+FTBCS approach for ${ }^{148-154} \mathrm{Sm}$

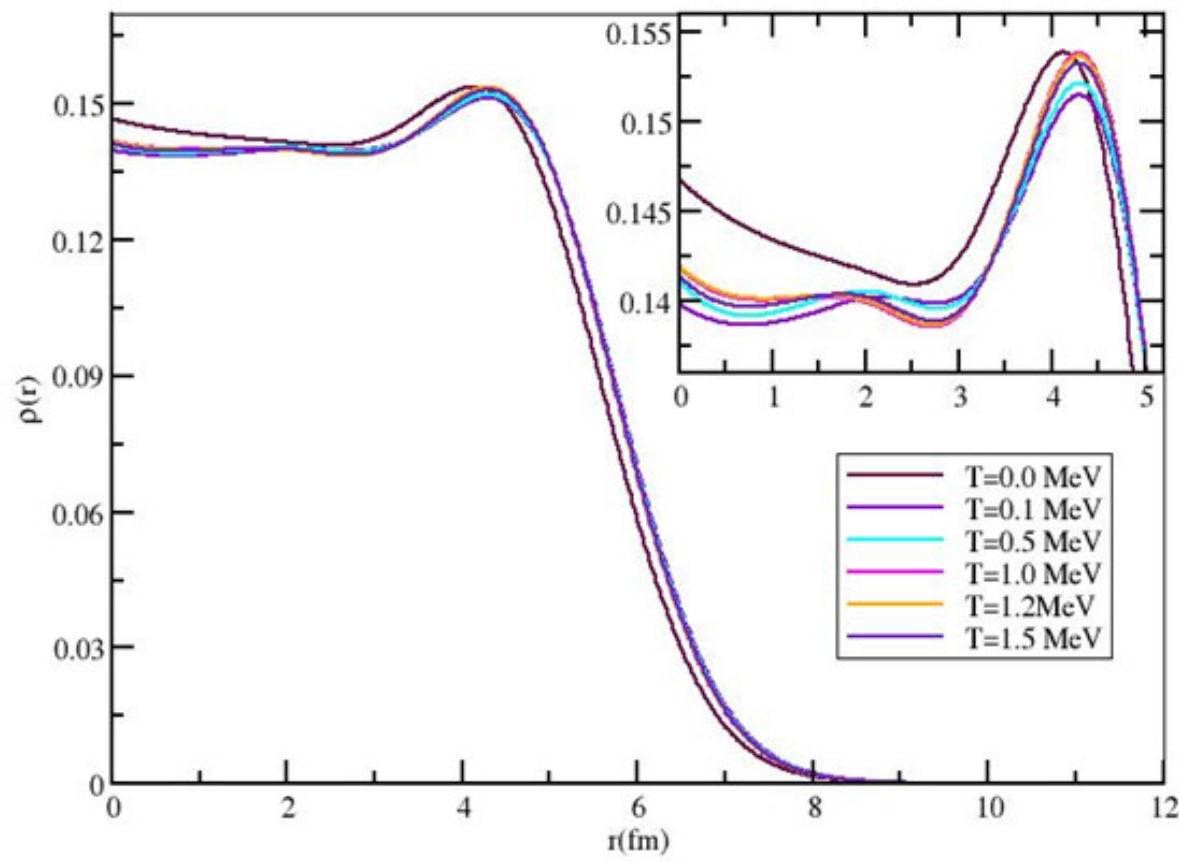

Figure 5. Variation of nuclear density profile with temperature for $\mathrm{T}=0.0$ to $2.0 \mathrm{MeV}$ using $\mathrm{RMF}\left(\mathrm{NL} 3^{*}\right)+\mathrm{FTBCS}$ approach for ${ }^{150} \mathrm{Sm}$. 
Figure 2 shows the temperature dependent variation of pairing energy $E_{\text {pair }}$ with temperature for $\mathrm{T}=0.0$ to $2.0 \mathrm{MeV}$ in $\mathrm{RMF}(\mathrm{NL} 3 *$ ) FTBCS approach. We can see that the pairing energy show a gradual decreasing trend with temperature. This decreasing trend of pairing energy with temperature confirms the destruction of superconducting behavior of nuclei. Moreover, one can infer that interactions between nucleons becomes weaker at some finite value of temperature i.e. at the critical temperature $T_{c}$ of nuclei, and there Coopers pairs are destroyed. It can also be seen that the pairing energy also decreases with increasing mass number(A). This is either due to closeness of shells or lesser probability of finding nucleons in outer shells. Figure 3 shows the temperature variation of binding energy (B.E.) for ${ }^{148-154} \mathrm{Sm}$ Nuclei. The binding energy remains almost constant till the temperature reaches at the critical value $T_{c}$ of the nuclei, and from there the binding energy falls gradually with temperature, which is very natural. One can notice that the decreasing nature in the binding energy curve at $T_{c}$ support the fact that nucleons become less bound as temperature increases. The gradual fall of curve at critical temperature shows that nucleons will become free at very high temperature.

Figure 4 show the variation of quadrupole deformation parameter $\beta_{2}$ of ${ }^{148-154} \mathrm{Sm}$ nuclei with temperature. The variation in the deformation parameter imply the shape transition in nuclei with temperature. As the temperature increases, the value of deformation parameter deviates from its ground state value at $T=0$. Initially, it increases to some higher value with an increase in temperature and later decreases with temperature. The deformation trend in all the $\mathrm{Sm}$ isotopes are same. In all the cases, with the temperature the nuclei moves towards higher prolate deformation and then comes down to lower prolate deformation. Finally,we can say that at very high value of temperature, the nuclei seems to be going towards the spherical in shape, which confirms the shape transition with temperature. Also, most of the studies confirm that nuclei tends to be spherical in shape at $\mathrm{T}>3 \mathrm{MeV}[50]$. So, at high value of temperature ${ }^{148-152} \mathrm{Sm}$ tends to spherical in shape. We have also calculated the temperature variation of the nuclear density using $\mathrm{RMF}(\mathrm{NL} 3 *)+\mathrm{FTBCS}$ model for $\mathrm{T}=0.0 \mathrm{MeV}$ to $2.0 \mathrm{MeV}$. The variation in density profile for all the isotopes seems to be similar in nature. So, we have presented here the temperature variation of the density profile for only ${ }^{150} \mathrm{Sm}$ nuclei. Figure 5 show the density profile of ${ }^{150} \mathrm{Sm}$ nuclei at different values of temperature. It is clear from the density profile that, the density values varies with temperature. The nuclear density which depends on spatial coordinates only, represents the distribution of nucleonic matter. In the present case, the variation shows that superconductivity play a central role. The central density also depends on pairing correlations. The paring correlation is destroyed with temperature and the density profile deviates from its ground state profile. The deviations in central density is most prominent and is clearly seen in Figure 5.

\section{CONCLUSIONS}

The temperature variation of the nuclear binding energy (B.E.), quadrupole deformation parameter $\beta_{2}$, neutron gap parameter $\left(\Delta_{n}\right)$, proton gap parameter $\left(\Delta_{p}\right)$, pairing energy $\left(\mathrm{E}_{\mathrm{pair}}\right)$ and the nuclear density profile have been studied within the RMF+FTBCS model using NL3* parameter set. The results obtained at zero temperature were found to be consistent with earlier calculations and the experimental values wherever available. The other important part of the work was introduction of the finite temperature in pairing correlations in RMF model i.e., FTBCS approach which is easily observable in nuclear parameters. We have calculated both the neutron and proton gap parameter as a function of temperature. The gap parameter tends to zero at a certain finite value of temperature known as critical temperature $T_{c}$. At this value, pairing phase transition takes place i.e., from super fluid state to normal state. Similarly, the pairing energy $\left(\mathrm{E}_{\text {pair }}\right)$ 
International Journal of Recent advances in Physics (IJRAP) Vol.4, No.4, November 2015

decrease with temperature due to destruction of the superconducting behavior of nuclei. Inference one can made that nucleons interaction weakens at finite value of temperature and Coopers pairs are destroyed. The decrease in binding energy reveals that nuclei are at excited state, and that the nucleons become loosely bound with the rise in temperature. The gradual fall of the curve at critical temperature shows that nucleons will become free at very high temperature. The variation in deformation parameter confirms the shape transition in ${ }^{148-154} \mathrm{Sm}$ nuclei. The nuclear density profile also varies with temperature. In the present case, the variation shows that superconductivity play a central role. The central density also depends on pairing correlations. As with the increase in temperature the paring correlation is destroyed and the density profile deviates from its ground state profile which, increase with temperature.

\section{REFERENCES}

[1] J. Bardeen, L .N. Cooper, and J. R. Schrieffer, Phys. Rev. 108 (1957) 1175.

[2] A. Bohr, B.R. Mottelson, and D. Pines, Phys. Rev. 110 (1958) 936.

[3] A. Bohr, and B.R. Mottelson, Nuclear Structure (W.A. Benjamin, New York, 1975) Vol.2.

[4] P. Ring, and P. Schuck, The Nuclear Many Body Problems (Springer, New York,2004).

[5] M. Fellah, T. F. Hammann, and D.E. Medjadi, Phys. Rev C 8 (1973) 1585.

[6] M. R. Oudih, M. Fellah, and N. H. Allal, Int. J. Mod. Phys. E 12 (2003) 109.

[7] M. R. Oudih, M. Fellah, N. H. Allal, and N. Benhamouda, Int. J. Mod. Phys. E 15 (2006) 643.

[8] N. H. Allal, M. Fellah, M. R. Oudih, and N. Benhamouda, Eur. Phys. J. A 27(2006) 301.

[9] M. R. Oudih, M. Fellah, N. H. Allal, and N. Benhamouda, Phys. Rev. C 76 (2007) 047307.

[10] N. Benhamouda, M. R. Oudih, N. H. Allal, and M. Fellah, Nucl. Phys. A 690 (2001) 219.

[11] N. Benhamouda, N. H. Allal, M. Fellah, and M. R. Oudih, Int. J. Mod. Phys. E 14 (2005) 197.

[12] N. H. Allal, and M. Fellah, Phys. Rev. C 43 (1991) 2648.

[13] A. L. Goodman, Phys. Rev. C 29 (1984) 1887.

[14] P. Bonche, S. D. Levit, and D. Vutherian, Nucl. Phys. A 436 (1985) 265.

[15] M. Brack, and P. Quentin, Nucl. Phys. A 361 (1981) 35.

[16] J. Rekstad et al., Phys. Lett. 117 B (1982) 384.

[17] A. L. Goodman, Nucl. Phys. A 352 (1981) 30.

[18] A. L. Goodman, Phys. Rev. C 38 (1988) 1092.

[19] M. Sano, and S. Yamazaki, Prog. Theor. Phys. 29 (1963) 397.

[20] L. G. Moretto, Nucl. Phys. A 185 (1972) 145.

[21] F. Alasia, O. Civitarese, and M. Reboiro, Phys. Rev. C 35 (1987) 812.

[22] C. Esebbag, and J. L. Edigo, Nucl. Phys. A.552 (1953) 205.

[23] N. Sandulescu, O. Civitarese, and R. J. Liotta, Phys. Rev. C 61 (2000) 044317.

[24] B. D. Serot, and J. D. Walecka, Adv. Nucl. Phys. 16 (1986) 1.

[25] W. Pannert, P. Ring, and J. Boguta, Phys. Rev. Lett. 59 (1986) 2420.

[26] Y. K. Gambhir, P. Ring, and A. Thimet, Ann. Phys. (N.Y.) 198 (1990) 132.

[27] A. Makishima, M. Adachi, H. Taketani, and M. Ishii, Phys. Rev. C 34 (1986)576.

[28] T. Klung et al., Phys. Lett. 495 B (2000) 55.

[29] E. Veje et al., Nucl. Phys. A 109 (1968) 489.

[30] P. Regan et al., Phys. Rev. C 51 (1995) 1745.

[31] W. Starzecki et al., Phys. Lett. 200 B (1988) 419.

[32] A. Bruce et al., Phys. Rev. C 50 (1994) 480.

[33] Abood N Saad et al., Eur. Jour. of Acad. Essays 1(3) (2014)76.

[34] G. A. Lalazissis et al., Phys. Lett. 671B (2009) 36.

[35] G. A. Lalazissis et al., Phys. Rev. C 55 (1997) 540.

[36] T. Niksic, D. Vretenar, P. Finelli, and P. Ring, Phys. Rev. C 66 (2002) 024306. 